\title{
Eventos Estressantes, Estratégias de Enfrentamento, Auto-Eficácia e Sintomas Depressivos entre Idosos Residentes na Comunidade
}

\author{
Stressful Events, Coping Strategies, Self-Efficacy and Depressive Symptoms Among \\ the Elderly Residing in the Community
}

\author{
Andréa Cristina Garofe Fortes-Burgos ${ }^{a}$, Anita Liberalesso Neri ${ }^{* a}$ \\ $\&$ Ana Paula Fabrino Bretas Cupertino ${ }^{b}$ \\ ${ }^{a}$ Universidade Estadual de Campinas, Campinas, Brasil \\ ${ }^{b}$ University of Kansas, Lawrence, Estados Unidos
}

\begin{abstract}
Resumo
Foram investigadas relações entre eventos de vida estressantes, estratégias de enfrentamento, auto-eficácia no enfrentamento e depressão em 544 participantes de um estudo sobre envelhecimento bem-sucedido com idosos residentes na comunidade $(74,6 \%$ mulheres; idade $=72,11, \pm 8.29 ; 42,1 \%$ de 60 a 69 anos; $39 \%$, de 70 a 79 e $18,9 \%, 80$ a 99 ). Os eventos estressantes foram classificados nas categorias finitude, problemas dos filhos, cuidado, crise e bem-estar psicológico. As estratégias de enfrentamento geraram cinco fatores: emoções negativas, controle ambiental, religiosidade, esquiva, inibição das emoções. Auto-eficácia no enfrentamento foi avaliada como adequada x inadequada. Na CES-D, o escore médio foi $10.24( \pm 8.66)$, para nota de corte $>11$, e a prevalência $32 \%$. Maior risco para depressão foi associado a emoções negativas, eventos incontroláveis, ter de 60 a 69 anos e avaliar a auto-eficácia como inadequada. Os eventos estressantes foram menos preditivos de depressão do que a avaliação cognitiva e o enfrentamento.

Palavras-chave: Eventos de vida estressantes; enfrentamento; auto-eficácia; depressão; idosos.
\end{abstract}

\begin{abstract}
The relationship among stressful life events, coping strategies, self-efficacy in coping and depression in 544 participants of a study on successful aging involving elderly who reside in the community was assessed $(74,6 \%$ women; age $=72.11 ; \pm 8.29 ; 42,1 \%$ age $60-69 ; 39 \% 70-79$ and $18,9 \%$ 80-99). Stressful life events were classified into categories related to: finitude, problems concerning offspring, care, crisis and psychological well-being. Coping strategies generated five factors: negative emotions, environment control, religiosity, avoidance behaviors, and emotional inhibition. Self-efficacy of coping was evaluated between appropriate versus inappropriate. Mean score of depression assessed through CES-D was 10.24, $\pm 8,66$ ); prevalence was $32 \%$ to a cut-off score $>11$. Major risk for depression was associated with negative emotions, uncontrollable events, age 60-69 and inappropiate self-efficacy of coping. The stressful life events seemed less predictive of depression than the cognitive assessment and the coping did.

Keywords: Stressful life events; coping; self-efficacy; depression; elderly.
\end{abstract}

As mudanças que acontecem ao longo das trajetórias de envelhecimento e desenvolvimento podem ser analisadas em termos de suas relações com eventos de vida, que oferecem opor tunidades para que elas ocorram. Eventos de vida são, assim, fatos de origem biológica, psicológica, social, sócio-cultural e ecológica que ocorrem ao longo da vida dos indivíduos e que organizam a sua experiência de desenvolvimento. Marcam a trajetória das pessoas, a passagem de um status evolutivo para outro, a passagem do tempo individual e do tempo histórico, o alcance de metas e o cumprimento de expectativas individuais e sociais. Eles orientam a

\footnotetext{
* Endereço para correspondência: Universidade Estadual de Campinas, Faculdade de Educação, Departamento de Psicologia Educacional, Av. Bertrand Russell, 801, Barão Geraldo, Campinas, SP, 13083-970. Tel.: (19) 3788-5555; Fax: (19) 3788-5573. E-mail: anitalbn@fcm.unicamp.br, geron@fcm.unicamp.br
}

personalidade rumo ao enfrentamento, pois são situações que, de alguma forma, desafiam o ajustamento biológico, social e psicológico (Aldwin \& Gilmer, 2004; Davies, 1996).

A ocorrência de eventos estressantes por si só não determina conseqüências negativas para as pessoas. As conseqüências negativas, positivas ou neutras desses eventos para bem-estar dependem de avaliação cognitiva sobre a natureza e sobre a demanda que tais eventos exercem sobre os recursos pessoais e sociais, e também das estratégias de enfrentamento que são capazes de acionar. Entre os idosos, há expressiva variabilidade na experiência dos eventos estressantes e nas formas de enfrentamento, uma vez que estão expostos a diferentes circunstâncias sociais e pessoais, atuais e passadas, além de serem dotados de diferentes graus de resiliência e possuírem diferentes formas de interpretar e lidar com eventos estressantes (Aldwin, 1994; Aldwin \& Gilmer, 2004). Esta condição depende em 
grande parte dos mecanismos de auto-regulação do self, entre os quais as estratégias de enfrentamento e o senso de auto-eficácia relacionado ao enfrentamento (Bandura, 1977, 1986, 1995, 1997).

Segundo Aldwin (1994), o "estresse refere-se a uma determinada qualidade de experiência, produzida por meio da interação entre o indivíduo e o ambiente, a qual, quer mediante super-ativação, quer mediante rebaixamento do nível de alerta, resulta em desconfor to psicológico ou fisiológico" (p. 22). A definição de Aldwin é consistente com a perspectiva interacionista de Lazarus e Folkman (1984, 1987), que conceituam o fenômeno do estresse considerando diferentes partes ou componentes: de um lado o tipo de experiência capaz de produzir estresse e de outro as reações individuais à tal experiência, incluindo reações fisiológicas e emocionais e mecanismos cognitivos de avaliação, atuando em interação recíproca. Estes autores definiram estratégias de enfrentamento como "os esforços cognitivos e comportamentais, em constante mudança, para lidar com mudanças internas e externas, avaliadas como superiores aos recursos pessoais" (1984, p. 141) e classificaram-nas em duas categorias: (a) enfrentamento baseado no problema, cujo foco é a ação do indivíduo sobre o meio, visando a modificar o ambiente ou seja, reduzir o efeito do evento estressor, e (b) enfrentamento baseado na emoção ou na cognição, cujo foco seria a modificação do indivíduo, por meio da regulação do desgaste emocional provocado pela situação de estresse.

Lazarus e Folkman (1987) submeteram o seu paradigma à prova mediante a realização de análises fatoriais sobre os escores obtidos com a aplicação da escala Ways of Coping. Resultou uma estrutura contendo oito fatores, que têm como variável latente as estratégias focalizadas no problema x na emoção: enfrentamento confrontativo, aceitação de responsabilidade, solução planejada do problema, distanciamento, auto-controle, busca de suporte social, fuga-esquiva, e reavaliação positiva. Stone, Helder e Schneider (1988) reconheceram sete fatores para as estratégias de enfrentamento: suporte social, busca de informações, religiosidade, redefinição da situação, evitação ou afastamento, redução da tensão e solução do problema. Aldwin e Gilmer (2004) apontaram os seguintes fatores: enfrentamento focalizado no problema, enfrentamento focalizado da emoção, enfrentamento através do suporte social, enfrentamento religioso e enfrentamento ligado à busca de um significado para o problema. Modelos estatísticos permitem a identificação de grupos ou de fatores correspondentes às estratégias de enfrentamento. Um dos objetivos do presente estudo é confrontar as definições teóricas existentes na literatura com soluções estatísticas geradas a partir de dados nacionais sobre as estratégias de enfrentamento do estresse entre idosos.

A avaliação do potencial estressor de um evento e o acionamento de estratégias de enfrentamento dependem dos mecanismos psicológicos de auto-regulação do self, entre os quais o senso de auto-eficácia, que se refere a crenças nas próprias capacidades para organizar e executar cursos de comportamentos necessários para lidar com situa- ções prospectivas envolvendo elementos estressantes, ambíguos e imprevisíveis (Bandura, 1977, 1982, 1997). As crenças de auto-eficácia influenciam os pensamentos, os sentimentos, a auto-motivação e a ação (Bandura, 1995), e podem determinar a decisão pessoal quanto ao início de um comportamento de enfrentamento, o seu tempo de duração e a quantidade de esforço desprendido em tal comportamento. Estudos recentes têm considerado as expectativas de auto-eficácia como parte integrante do processo de estresse (O'Leary, 1992), particularmente a sua influência sobre os processos de avaliação e enfrentamento (Karademas \& Kalantzi-Azizi, 2003).

Pesquisas sobre as diferenças de idade quanto à ocorrência de eventos de vida revelaram que os idosos vivem mais experiências de doenças com hospitalização, aposentadoria e morte de cônjuge ou de entes queridos, enquanto que adultos jovens vivem mais eventos relacionados ao trabalho, à situação financeira e à vida familiar (Aldwin, Sutton \& Lachman, 1996; Folkman, Lazarus, Pimley \& Novacek, 1987; Hughes, Blazer \& George, 1988).

\section{Objetivos}

A presente investigação teve como objetivo analisar a relação entre experiência de eventos estressantes e sintomas depressivos em mulheres e homens idosos pertencentes a três grupos de idade, relação essa mediada por estratégias de enfrentamento e pela avaliação da auto-eficácia no uso dessas estratégias.

\section{Método}

\section{Participantes}

Os idosos integravam o Projeto Estudo do Envelhecimento Saudável (PENSA) ${ }^{1}$, para o qual foram selecionados pelo método de amostragem sistemática. Para tanto, foram visitados todos os domicílios dos bairros da cidade de Juiz de Fora, $\mathrm{MG}^{2}$, cujo percentual de idosos (60 anos e mais) residentes era superior a 15\% (14 bairros, incluindo o Centro), segundo dados do Censo 2000 do Instituto Brasileiro de Geografia e Estatística ([IBGE], 2000). Recrutadores treinados foram de porta-em-porta para identificar residências que tivessem pelo menos um morador idoso em condições físicas e cognitivas e que quisesse responder aos instrumentos da pesquisa. No total, foram visitados 7089 domicílios, dentre os quais foram identificados 1686 com pelo menos um idoso. Novecentos e cinqüenta e seis idosos aceitaram participar do estudo (56\%), 614 recusaram $(36 \%)$ e $116(8 \%)$ pareceram física ou cognitivamente incapacitados. Todos os idosos que aceitaram participar do estudo preencheram uma ficha de re-

\footnotetext{
${ }^{1}$ Inicialmente financiado pelo CNPq, programa de fixação de doutores - PROFIX, e aprovado pelo comitê de ética em pesquisa da Universidade Federal de Juiz de Fora. Amostra total de 956 idosos

2 A cidade de Juiz de Fora, tem aproximadamente 436.432 habitantes, sendo 10,6\% idosos (acima de 60 anos). Este índice é superior a população idosa do estado de Minas Gerais $(9,8 \%)$ e do Brasil ( $8,6 \%)$
} 
crutamento e assinavam um Termo de Consentimento Livre e Esclarecido. Cada entrevista foi realizada numa única sessão, de aproximadamente duas horas, envolvendo itens de auto-relato, de natureza sócio-demográfica, física, psicológica e sociológica. Os dados foram coletados entre 2002 a 2003. As respostas eram anotadas num protocolo e depois inseridas num banco de dados.

Para o presente estudo, foram selecionados 544 dentre os 956 participantes do PENSA. O critério de seleção da amostra foi terem relatado que viveram eventos estressantes do curso de vida nos últimos cinco anos. Dos 544 idosos, 74,6\% eram mulheres, com idade média de 72,1 anos $(D P=8.29) ; 42,1 \%$ tinham entre 60 e 69 anos e $39 \%$ entre 70 e 79 anos e $18,9 \%$ tinham 80 anos e mais. Cinqüenta por cento eram casados, $39,5 \%$ viúvos, $6,25 \%$ solteiros e 4,6\% divorciados, desquitados ou separados. Metade dos idosos era alfabetizada ou tinha o curso primário, outros $17 \%$ tinham o ginasial e $21 \%$ o curso colegial. Dez por cento tinham curso superior, completo ou incompleto.

\section{Materiais}

Três instrumentos de autoria de Aldwin et al. (1996) foram traduzidos e adaptados semanticamente da língua inglesa para a língua portuguesa, para efeito do uso na pesquisa: o Inventario de Eventos Estressantes, a Avaliação da Intensidade de um Evento de Vida Negativo e o Inventário de Enfrentamento da Califórnia. Para a avaliação do senso de auto-eficácia do enfrentamento foi elaborado um instrumento especifico. Além destes, foi aplicada a Center Epidemiologic Survey - Depression [CES-D] (Radloff, 1977), validada para o português (Tavares, 2004, Tavares-Batistoni, Neri \& Cupertino, 2006).

1. Inventário de Eventos Estressantes: Consiste em duas listas de eventos estressantes do curso de vida. A primeira lista de eventos corresponde a situações positivas, a qual não foi utilizada por este estudo. A segunda lista é composta por 31 itens que descrevem eventos negativos, tais como problemas de saúde ou nas relações conjugais e morte dos pais, e foi utilizada nesta pesquisa. Os participantes indicam quais dos eventos listados estiveram presentes em sua vida e mencionam a época em que ocorreram - se no último ano, nos últimos cinco anos, há mais de cinco anos, ou se não aconteceram.

2. Avaliação da intensidade de um evento de vida negativo: Depois de responderem ao inventário de eventos de vida negativos, a pesquisa solicitava que cada participante apontasse um evento que, a seu ver, tinha sido o mais estressante de todos os que havia apontado. Então, era introduzida uma questão que solicitava avaliação pessoal do impacto desse evento estressante: "Em uma escala de 1 a 7 , que nota daria para este evento em termos de ser estressante?" (sendo 1 = "nada estressante" e 7 = "muito estressante"). Quando o participante escolhia apenas um evento, era esse que avaliava a partir da pergunta. É importante ressaltar que as questões sobre o enfrentamento do estresse, a avaliação da auto-eficácia e a avaliação da intensidade do estresse, foram respondidas com relação a um único evento negativo que cada participante apontou como o mais estressante vivido nos últimos cinco anos.

3. Estratégias de enfrentamento (coping): Foi utilizado o Inventário de Enfrentamento da Califórnia (Aldwin et al., 1996). Consiste em 19 questões escalares de quatro pontos cada uma, indicativas da freqüência do uso das estratégias focadas no problema ou na emoção. Era solicitado que os idosos apontassem a freqüência com que utilizaram as estratégias contidas no inventário. Para efeito do tratamento, a escala foi considerada como dicotômica ( $\operatorname{sim} x$ não).

4. Percepção de auto-eficácia no enfrentamento: Foram duas questões relacionadas ao enfrentamento do evento negativo: "Dadas estas circunstâncias, como diria que lidou com o problema?" e "Quão bem diria que lidaria com um problema como este no futuro?”. As respostas eram referenciadas a uma escala de 1 a 5 , sendo 1 = "nada bem, ou seja, muito mal" e 5 = "muito bem".

5. Sintomas depressivos: Para avaliar estados depressivos foi utilizada a escala CES-D (Center Epidemiologic Survey - Depression), validada no Brasil para a população idosa (Tavares, 2004). A escala original foi desenvolvida por Radloff (1977), no National Institute of Mental Health dos Estados Unidos, e tem como finalidade detectar estados depressivos em populações adultas, por meio do auto-relato de sintomas ligados à depressão. A CES-D permite classificar os indivíduos como deprimidos e não-deprimidos, mediante uma nota de corte que, no Brasil, o estudo de Tavares estabeleceu ser $>11$. Esta nota foi obtida com base em comparação com o comportamento dos mesmos indivíduos na Geriatric Depression Scale (GDS), de Yesavage et al. (1983), validada no Brasil por Almeida e Almeida (1999).

\section{Resultados}

Foram feitas análises univariadas e multivariadas, primeiro focalizando o estabelecimento de tendências gerais e depois a busca de padrões de relações entre as variáveis.

\section{Eventos Estressantes}

Os eventos de vida estressantes foram submetidos à análise de conteúdo, mediante a qual foram derivadas categorias. Foi feita análise freqüência de cada uma delas segundo critérios de gênero e de idade. As categorias foram:

1.Eventos relacionados à finitude: morte de pessoas amadas ou problemas de saúde da própria pessoa ou de alguém próximo, que representam ameaça à continuidade da vida.

2. Eventos que afetam os descendentes: ocorrências cujo curso ou cujos efeitos não podem ser alterados pelo idoso, porque dependem de circunstâncias ligadas à vida de outrem. Geram senso de incontrolabilidade e desconforto em parte por causa do apego afetivo que 
une membros da família, em parte porque algumas dizem respeito ao desempenho de papéis sociais cujo adequado cumprimento pelos descendentes confirma os esforços educativos dos pais.

3. Eventos relacionados ao cuidado: pressões externas (geradas pelo curso da doença, pelo aumento de despesas, por conflitos familiares e pela competição do cuidado com papéis profissionais e familiares) ou internas (tristeza, ressentimento, compaixão, medo, dúvida) geradas pelo exercício do cuidado prestado a um outro adul to significativo para o idoso e que fragilizado.

4. Eventos que afetam o bem-estar psicológico: relacionados ao ajustamento pessoal na velhice expressos em termos de ameaça ao senso de significado, sentimentos de solidão, problemas de saúde mental, reais ou presumidos, e crise espiritual.

5. Eventos pontuais de crise: Marcam mudanças normativas (por exemplo a aposentadoria e o empobrecimento) ou não-normativas no curso do envelhecimento (por exemplo o divórcio).
Os eventos relacionados à finitude foram os mais relatados $(66 \%$ dos idosos), seguidos de eventos que afetam a vida dos descendentes (19\%), eventos pontuais $(8 \%)$, relacionados ao bem-estar (4\%) e ao cuidado (3\%). Os idosos avaliaram os eventos vividos como muito estressantes $(M=$ 5.94; Mediana $=7 ; D P=1.49$ ). As mulheres avaliaram mais os eventos como extremamente estressantes do que os homens (teste Qui-quadrado: $\chi^{2}=8.91$; GL $=2 ; p=0.012$ ). Porém, as comparações entre os grupos etários não revelaram diferença estatisticamente significante (teste qui-quadrado: $\left.\chi^{2}=1.10 ; \mathrm{GL}=4 ; p=0.894\right)$.

\section{Estratégias de Enfrentamento}

Foi realizada análise fatorial (método de componentes principais e rotação Varimax) para os itens do Inventário de Estratégias de Enfrentamento. O índice Kaiser-MeierOlkin (KMO) foi de 0,5935, indicando consistência intermediária. Foram obtidos cinco fatores com valores próprios superiores a 1 e que explicaram $43,6 \%$ da variabilidade total da distribuição.

Tabela 1

Estrutura Fatorial obtida para a Escala de Estratégias de Enfrentamento

\begin{tabular}{lll}
\hline Fatores & Cargas & Itens com maior carga no fator \\
\hline Fator 1 & 0.612 & E7 - Demonstrou hostilidade aos outros \\
& 0.598 & E9 - Se isolou dos outros \\
& 0.452 & E18 - Usou remédios para controlar ansiedade ou depressão \\
& 0.429 & E19 - Gritou e xingou \\
& 0.343 & E13 - Usou comida, drogas ou álcool para escapar da situação \\
& -0.525 & E1 - Aceitou que era um problema que tinha que enfrentar (carga invertida) \\
Fator 2 & 0.697 & E10 - Confiou que os outros fariam a coisa certa \\
& 0.603 & E5 - Esperou para decidir/agir até ter mais informações \\
& 0.532 & E16 - Parou suas atividades quando precisou \\
Fator 3 & 0.452 & E14 - Fortaleceu seus laços com os demais \\
& 0.853 & E15 - Confiou em Deus ou algum outro ser superior \\
Fator 4 & 0.801 & E17 - Tentou esquecer que o problema existia \\
& 0.655 & E6 - Procurou distrair-se com livros, hobbies, TV \\
& 0.643 & E12 - Chegou à conclusão de que as coisas poderiam ter sido piores \\
& 0.504 & E4 - Decidiu que não havia nada a ser feito \\
Fator 5 & 0.430 & E11 - Guardou seus sentimentos para si mesmo \\
& 0.690 & E2 - Culpou outros (carga invertida) \\
\hline
\end{tabular}

Para interpretar a estrutura fatorial obtida, tomamos como referência a tipologia de Folkman e Lazarus (1980). Três fatores tiveram foco no controle da emoção (1, 3 e 4) e um na solução do problema (fator 2). As denominações dos fatores foram as seguintes: (a) Fator 1. Foco na expressão de emoções negativas, em excessos comportamentais e em comportamentos de risco. Permitem à pessoa esquivar-se de culpa, de ressentimento e da dúvida, lidar com ansiedade e depressão e, de alguma forma, entrar em contato com os outros, quer para acionar ajuda, quer manter a ativação emocional; (b) Fator 2. Foco em tentativas de controle sobre o ambiente. Acreditar que os outros darão conta do evento e assim permitir que lidem com ele, tentar obter mais informações antes de agir, parar as atividades quando necessário e procurar suporte social são ações que refletem uma postura pró-ativa, mediada por ações dos semelhantes, que o indivíduo é capaz de evocar. Envolvem tentativas de controle indireto sobre o ambien- 
te; (c) Fator 3. Foco na religiosidade como elemento amortecedor. Ações voltadas para o controle emocional e para a mudança de crenças, visando à regulação das emoções e à proteção do self, em situações de incontrolabilidade; (d) Fator 4. Foco na atenuação do potencial estressor do evento, mediante comportamentos de esquiva. Este padrão tem relação com mecanismos cognitivos que atenuam o potencial estressor do evento e sem expor a pessoa a conseqüências adversas, contribuem para diminuir a depressão e a ansiedade; (e) Fator 5. Foco em inibição das emoções. Tendência à não-expressão emocional aberta e ao recolhimento ao mundo interno.

Os itens da Escala de Enfrentamento foram submetidos à análise de consistência interna. O alfa obtido foi de 0,520. Os alfas para os itens dos fatores foram Fator $1: \mathrm{a}=0.486$; Fator 2: $\mathrm{a}=0.493$; Fator 3: $\mathrm{a}=0.675$; Fator 4: $\mathrm{a}=0.403$; Fator 5: $\mathrm{a}=0.250$. Embora o valor de alfa abaixo de 0.60 seja considerado fraco, a literatura sobre estratégias de enfrentamento registra baixos índices de consistência interna, justamente porque as pessoas não usam somente um tipo de estratégia, uma vez que elas não são disposições gerais para o compor tamento mas têm seu uso determinado pela situação. Segundo Folkman (1992), os pesquisadores devem usar critérios menos exigentes na avaliação da confiabilidade de medidas de enfrentamento (um alfa igual a 0.60 pode ser apropriado), visto que dificilmente as estratégias possuem uma natureza unidimensional. Mais do que isso, afirma ela, independentemente do nível de consistência interna que um pesquisador está disposto a aceitar, ele não deve fiar-se em valores previamente estabelecidos, mas sim avaliar e relatar o nível obtido em sua investigação.

Análises comparativas entre grupos de gênero e idade em relação aos tipos de estratégias de enfrentamento revelaram diferenças estatisticamente significantes (teste $U$ de Mann-Whitney). Houve maior presença de estratégias ligadas à religiosidade nas mulheres e de estratégias relacionadas à inibição da emoção nos homens; e maior presença de estratégias associadas à intensificação de emoções negativas, excessos compor tamentais e comportamentos de risco entre os idosos mais novos ( $<7 \mathrm{O}$ anos).

\section{Relações entre a Experiência de Eventos Estressantes e as Estratégias de Enfrentamento}

Os dados foram submetidos à análise de agrupamentos (Pereira, 1999; Vogt, 1993). Análises inferenciais utilizadas para conhecer os desempenhos dos diferentes grupos de sujeitos por critérios de gênero e idade resultaram em valores estatisticamente significantes para gênero (teste Qui-quadrado: $\chi^{2}=31.97$; GL $=3 ; p<0.001$ ); para idade (teste Qui-quadrado: $\chi^{2}=75.42$; GL $=6 ; p<0.001$ ); para eventos estressantes (teste Qui-quadrado: $\chi^{2}=525.7$; GL $=12 ; p<0.001$ ) e para estratégias (teste Kruskal-Wallis: fator 1 ( $p=0.003)$, fator $2(p<0.001)$, fator $3(p<0.001)$, fator $4(p<0.001)$ e fator $5(p<0.001)$.

A análise de agrupamentos resultou nos seguintes grupos: (a) Homens de 60 a 79 anos, que passaram por eventos de finitude e por eventos que afetam a descendência, que relataram recorrer menos à religiosidade e que são mais propensos a guardar seus sentimentos, em vez de procurar outras pessoas para desabafar; (b) Mulheres de 70 anos e mais, que viveram eventos de finitude e que relataram ter utilizado estratégias ligadas à religiosidade; (c) Mulheres de 60 a 69 anos, que relataram ter cuidado de outro adulto, ter vivenciado problemas de saúde dos pais, ter se envolvido com questões ligadas ao próprio bemestar psicológico, ter vivido eventos de crise, utilizaram mais estratégias focalizadas na intensificação de emoções negativas, excessos comportamentais e comportamentos de risco; (d) Mulheres de 60 a 69 anos, que relataram ter vivido eventos de finitude utilizaram mais estratégias ligadas à religiosidade, estratégias focalizadas na solução do problema e estratégias de evitação mediante o acionamento de recursos pessoais.

\section{Senso de Auto-eficácia em Relação às Estratégias de Enfrentamento Adotadas}

As análises de freqüência para a amostra total $(N=544)$ revelaram que os idosos consideraram adequadas as formas de enfrentamento que adotaram $(M=3.14$; Mediana $=3 ; D P=1.15)$. As análises comparativas permitem concluir que não houve diferença estatisticamente significante com relação à avaliação da auto-eficácia segundo os critérios de gênero e idade (teste Qui-quadrado: $\chi^{2}=0.86$; $\mathrm{GL}=1 ; p=0.354$ e teste Qui-quadrado: $\chi^{2}=0.58$; $\mathrm{GL}=2$; $p=0.748$, respectivamente).

Relações entre Estresse, Enfrentamento e Sintomas Depressivos A partir da aplicação da CES-D foi possível identificar os indivíduos que apresentaram sintomas depressivos na semana precedente à entrevista (nota de corte $>11)$. Houve nesta amostra uma prevalência comparável (32\%) à da amostra total do PENSA, esta descrita por Tavares (2004) como superior à obtida em outros estudos populacionais que utilizaram a GDS. O escore médio da amostra $(N=$ 544) na CES-D foi 10,24, o $D P=8$,66 e a Mediana $=8$. As relações existentes entre sintomas depressivos, gênero, idade, eventos estressantes, estratégias de enfrentamento e auto-eficácia, permitiram as seguintes conclusões: (a) Homens e mulheres não diferiram de forma estatisticamente significante quanto à depressão (teste Qui-quadra$d o: \chi^{2}=0.35$; GL=1; $p=0.557$ ); (b) Os idosos de menos de 70 anos apresentaram mais sintomas depressivos em comparação com os de 70 a 79 anos e mais de 80 anos (teste Qui-quadrado: $\chi^{2}=10.05$; GL=2; $p=0.007$ ); (c) Predominaram eventos de finitude e pontuais entre os não deprimidos e eventos que afetam a descendência, ligados ao cuidado e ligados ao próprio bem-estar psicológico entre os deprimidos (teste Qui-quadrado: $\chi^{2}=17.28$; GL $=4 ; p$ = 0.002); (d) Não houve diferença estatisticamente significante com relação à freqüência do uso de estratégias pelos idosos não deprimidos. Idosos deprimidos pontuaram mais no fator 1 e menos no fator 2 (teste $U$ de MannWhitney: fator $1(p<0.001)$, fator 2 ( $p=0.227)$, fator 3 $(p<0.001)$, fator $4(p=0.861)$ e fator $5(p=0.556))$; (e) Houve mais idosos deprimidos entre os que apresentaram baixa eficácia percebida atual e futura (teste Qui-quadrado: $\chi^{2}$ 
= 23.45; $\mathrm{GL}=4 ; p<0.001$ e $\chi^{2}=20.32 ; \mathrm{GL}=4 ; p<$ 0.001, respectivamente); (f) Nos quatro agrupamentos revelados pela análise de cluster havia indivíduos deprimidos e não deprimidos, tanto que as diferenças não foram estatisticamente significantes (teste Qui-quadrado: $\chi^{2}=$ 4.83; $\mathrm{GL}=3 ; p=0.185$ )

$\mathrm{O}$ conjunto de dados foi submetido à análise de regressão pelo modelo multivariado, critério Stepwise de sele- ção de variáveis. Tiveram efeitos significativos sobre depressão, conjuntamente, as variáveis intensificação de emoções negativas, excessos compor tamentais e comportamentos de risco (fator 1), eventos que ameaçam o próprio bemestar e eventos que afetam a descendência, ter de 60 a 69 anos e ter baixa auto-eficácia percebida no manejo de eventos estressantes. A Tabela 2 apresenta os dados resultantes dessa análise.

Tabela 2

Resultados da Análise de Regressão Logística Multivariada para Depressão

\begin{tabular}{|c|c|c|c|c|c|}
\hline Passo / Variável & Níveis de Comparação* & p-valor & O.R. & IC/O.R. & $95 \%$ \\
\hline 1. Estratégias - Fator 1 & $>\mathrm{P} 75 /<=\mathrm{P} 75$ & $<0.001$ & 3.48 & 2.08 & -5.83 \\
\hline \multirow{4}{*}{ 2. Eventos Estressantes } & Descendência / Finitude & $<0.001$ & 2.59 & 1.48 & -4.52 \\
\hline & Cuidar / Finitude & 0.289 & 1.96 & 0.57 & -6.76 \\
\hline & Bem-Estar / Finitude & 0.053 & 3.34 & 0.99 & -11.33 \\
\hline & Pontuais / Finitude & 0,479 & 1,35 & 0.59 & -3.06 \\
\hline \multirow{2}{*}{ 3. Idade } & $<70$ anos / 70-79 anos & 0.007 & 1.96 & 1.21 & -3.20 \\
\hline & $\geq 80$ anos $/ 70-79$ anos & 0.595 & 1.19 & 0.63 & -2.27 \\
\hline 4. Eficácia Percebida Atual & Inadequada (1-2) / Adequada (3-5) & 0.026 & 1.74 & 1.07 & -2.81 \\
\hline
\end{tabular}

Notas. OR (Odds Ratio) = Razão de Risco para Estado Depressivo; (n=144 Sim e n=284 Não). IC $95 \%$ OR = Intervalo de $95 \%$ de Confiança para a Razão de Risco. Critério de Seleção: Stepwise. * nível de comparação / nível de referência

\section{Discussão}

Mais da metade dos idosos relataram problemas de saúde deles mesmos ou de pessoas próximas e a morte de entes queridos como eventos estressantes, resultados similares aos relatados por estudos anteriores (Aldwin, 1990, 1991; Aldwin et al., 1996; Beckett, Goldman, Weinstein, Lin \& Chuang, 2002; Chapleski, Kaczynski, Gerbi \& Lichtenberg, 2004; Folkman et al., 1987; Krause, 1986). A experiência real de finitude pode ser traduzida em termos de maior dependência dos outros, por circunstância de incapacidades ou problemas graves de saúde. Situações que afetam os descendentes, desafiam o senso de continuidade e integridade do self na velhice, quando os indivíduos percebem que muitos dos seus esforços educativos (como pais ou avós) falharam, ou ainda, pela empatia em relação aos filhos e netos.

Estudos anteriores (Aneshensel, 1992; Mirowsky \& Ross, 1995) revelaram a tendência feminina em avaliar o estresse dos eventos como sendo de alta intensidade, assim como no presente estudo. Culturalmente, as mulheres tendem a falar mais de seus problemas do que os homens, queixar-se mais facilmente de enfermidades e de outros problemas em geral, o que caracteriza o grupo feminino como aquele que vive mais intensamente o estresse. Elas podem de fato perceber os eventos como sendo mais estressantes, ou sentir os efeitos do estresse mais do que os homens, por terem menos recursos materiais, serem mais oneradas física e economicamente. Por outro lado, pode-se especular que as mulheres dessa geração terão tido muitas chances de viver situações de incontrolabilidade associadas a preconceitos e a práticas sociais discriminativas em relação às mulheres. A queixa pode funcionar como uma estratégia de sobrevivência, uma vez que com freqüência as reclamações são úteis para chamar a atenção, para ganhar controle secundário sobre o ambiente e para evitar mais punição.

Os homens relataram mais eventos pontuais como sendo estressantes, dentre os quais a aposentadoria, o divórcio, as perdas financeiras, entre outros. Os eventos pontuais são ocorrências normativas ou esperadas no curso da vida, cujo enfrentamento pode ser beneficiado por mecanismos de socialização antecipatória e aumento do senso de controle sobre o ambiente e as próprias emoções (Neugarten, 1968). O caráter previsível ou controlável dos eventos pontuais, vividos pelos homens deste estudo, pode explicar, em parte, porque estes avaliaram os eventos com menor intensidade de estresse.

Problemas com os descendentes podem afetar as mulheres no início da velhice e os homens na metade da velhice, sendo portanto, considerados eventos estressantes bem presentes no envelhecimento. Houve maior variabilidade entre os homens, comparando os dois grupos etários formados entre 60 e 79 anos. Já entre as mulheres, houve predomínio de eventos que afetam a descendência nessas duas faixas etárias, sugerindo a importância atribuída pelo gênero feminino aos problemas que afetam os filhos ou os netos. Martire, Stephens e Townsend (2000) examinaram o estresse em papéis centrais - como ser mãe, esposa ou profissional - entre mulheres de meia-idade. As mulheres que tiveram problemas com os filhos relataram mais conseqüências negativas associadas ao estresse, comparadas 
com aquelas que viveram situações estressantes associadas aos papéis de esposa ou de profissional.

Os idosos mais velhos (>80) foram mais afetados por problemas de saúde e morte de entes queridos e por situações ligadas ao bem-estar pessoal, como sentimentos de solidão, problemas de saúde mental, reais ou presumidos, e crise espiritual. Os dados sobre os diferentes tipos de eventos vividos em diferentes idades reforçam a noção de que a velhice não se traduz num período homogêneo do desenvolvimento, ou seja, ter 60 anos é diferente de ter 70 ou 80. Por essa razão, a pesquisa em Gerontologia deve con-siderar as várias etapas do processo de envelhecimento, e as particularidades presentes em cada uma delas. Segundo Rodin (1986), incontrolabilidade e estresse fazem parte deste período, bem como todas as mudanças que ocorrem ao longo do processo de envelhecimento. $\mathrm{Na}$ presente investigação, os homens mostraram que no início da velhice o que mais causa estresse são problemas pontuais, sobre os quais eles podem ter maior controle. À medida que envelhecem, predominam eventos que afetam a descendência e mais tarde, aqueles que afetam diretamente o bem-estar pessoal.

As estratégias com foco na religiosidade, utilizadas predominantemente pelas mulheres, servem como um amortecedor dos eventos negativos, como elementos protetores do self, ajudando principalmente em situações de alta incontrolabilidade, servindo como elemento facilitador da aceitação da falta de controle, e preservando o self dos impactos negativos do estresse. Diferentemente das mulheres, os homens neste estudo tenderam a guardar seus sentimentos para si mesmo, ao invés de buscar apoio em outros, como forma de enfrentar o estresse.

Os idosos de 60 a 69 anos utilizaram mais estratégias com foco na expressão de emoções negativas, em excessos comportamentais e em comportamentos de risco, em comparação com os idosos acima de 70 anos. Tal diferença justifica-se no fato de que os idosos mais novos expressam mais emoções do que os mais velhos (Carstensen, Gross \& Fung, 1998). Quando mais novos, têm mais competência adaptativa, e assim, podem expressar de maneira mais vigorosa e variada as suas emoções positivas e negativas. A maioria relatou satisfação pessoal com seu desempenho no enfrentamento de um evento estressante. Tal avaliação pode ter conseqüências positivas para os idosos dessa amostra, uma vez que um forte senso de auto-eficácia promove realização e bem-estar (Karademas \& Kalantzi-Azizi, 2003).

As análises comparativas não revelaram diferença estatisticamente significante entre homens e mulheres quanto à presença de sintomas depressivos, reforçando o argumento de Barefoot, Mortensen, Helms, Avlund e Schroll (2001) de que, com o envelhecimento, homens e mulheres tendem a apresentar níveis similares de depressão. A tendência à diminuição dos sintomas depressivos com o envelhecimento revelou-se também através das análises comparativas entre as diferentes idades. Os idosos mais novos (de 60 a 69 anos) apresentaram índices significantemente maiores de depressão, em comparação com os idosos acima dos 70 anos.
Os eventos mais relacionados com a presença de depressão foram os que afetam a descendência, os relacionados ao cuidado a outro adulto e eventos relacionados ao próprio bem-estar psicológico. Aspectos como a incontrolabilidade, as dificuldades em desempenhar tarefas de cuidado ou sentimentos de ameaça ao próprio bem-estar pessoal podem gerar angústia ou afetar o senso de significado pessoal, gerar sentimentos de solidão, problemas de saúde mental, ou mesmo crise espiritual, imprimindo um quadro sintomático depressivo.

Entre os idosos deprimidos (>11) predominaram estratégias de enfrentamento cujo foco foi o controle do ambiente e estratégias relacionadas à intensificação de emoções negativas, excessos comportamentais e comportamentos de risco. Avaliaram a auto-eficácia no enfrentamento como inadequada, mostrando-se insatisfeitos com o próprio desempenho na situação estressante que enfrentaram. Além disso, quando indagadas sobre eventos semelhantes no futuro, essas pessoas também esperam que o enfrentamento seja inadequado. Tais resultados são condizentes com as principais manifestações cognitivas e emocionais da depressão, como por exemplo, os padrões negativos de pensamento, traduzidos em visão negativa de si mesmo, do ambiente e do futuro (Young, Beck \& Weinberg, 1999). O pensamento pessimista do deprimido faz com que ele avalie-se como mais ineficaz do que realmente é, percebendo os obstáculos oferecidos pela vida como barreiras intransponíveis (Seligman, 1977). Resultados semelhantes foram encontrados em outros estudos, nos quais avaliações negativas de auto-eficácia foram relacionadas com sintomas de ansiedade e estresse, depressão, sintomas psicossomáticos, e pior bem-estar psicológico (Bandura, 1997; Holahan \& Holahan, 1987; Kavanagh, 1992; O'Leary, 1992).

Brown e Harris (1978) sugerem que a depressão em adultos surge como resultado de fatores sociais estressantes, tais como eventos de vida (perdas) ou dificuldades crônicas, combinadas com vulnerabilidade e outros fatores tais como desvantagens sociais, perdas em relacionamentos íntimos, eventos traumáticos precoces, perdas educacionais e na capacidade intelectual, história pessoal e familiar de doença depressiva. O presente estudo examinou a relação entre os eventos estressantes e a presença de sintomas depressivos, mediada por mecanismos psicológicos de avaliação e enfrentamento, através de análises multivariadas, principalmente análises comparativas, conforme descritas anteriormente. Os resultados obtidos mostraram que o maior risco para depressão foi associado com: uso de estratégias de enfrentamento com foco na expressão de emoções negativas, em excessos comportamentais e em compor tamentos de risco, experiência de eventos que afetam a descendência, ter entre 60 e 69 anos, e avaliar a auto-eficácia no enfrentamento como "inadequada".

O enfrentamento do estresse foi fator de maior influência para depressão do que os outros fatores investigados $(\mathrm{OR}=3.48 ; p<0.001)$. Isso significada que os idosos deprimidos são aqueles que adotaram como estratégias de enfrentamento do estresse comportamentos de risco ou 
excessos comportamentais (como uso de remédios para controlar ansiedade e depressão, abuso de substâncias drogas, álcool - e comida para de drogas), e expressões emocionais negativas contra os outros (como hostilidade e agressividade). Tais comportamentos, cujo foco está na expressão das emoções, podem ser úteis a curto prazo, no momento em que a pessoa está enfrentando uma situação estressante. Porém, a médio e longo prazo, investir nesse tipo de comportamento pode acarretar prejuízos à saúde física (no caso de abuso de substâncias, ou mesmo comida em excesso), problemas nos relacionamentos interpessoais, ou ainda, problemas emocionais (gerados por punição administrada pelos outros, ou por atitudes de isolamento e de não-aceitação do problema). Considerando que os eventos negativos relatados foram vividos nos últimos 5 anos, é possível que os efeitos do enfrentamento a médio prazo sejam expressos em sintomas depressivos no presente.

Este estudo mostrou a variabilidade das experiências de eventos estressantes no envelhecimento, particularmente entre as mulheres. Revelou que embora vivendo experiências que desafiam a integridade do self e o controle pessoal, avaliadas como estressantes, os idosos mostraram-se satisfeitos com a forma como enfrentaram as situações, o que sugere a manutenção do potencial adaptativo na velhice.

Os resultados sugerem que as formas de enfrentamento do estresse tiveram associação mais forte com sintomatologia depressiva na velhice do que os eventos em si. Porém, não desconsideram a importância dos eventos de vida estressantes sobre a saúde mental dos idosos. Aspectos individuais podem contribuir em maior grau para a manifestação de sintomas depressivos entre idosos do que os determinantes ambientais. Dentro de uma visão interacional, como a adotada neste trabalho, o estresse é fruto de constantes interações entre pessoa e ambiente, nas quais as demandas ambientais superam as capacidades individuais de enfrentamento. As conseqüências dessas interações podem ser tanto negativas - na forma de sintomas depressivos, por exemplo - ou positivas - na forma de estratégias de enfrentamento, por exemplo.

Algumas limitações metodológicas devem ser consideradas, como por exemplo, a configuração da amostra. Não se trata de uma amostra probabilística, uma vez que a amostra pertencente ao projeto PENSA foi selecionada pelo método de amostragem sistemática. Pode-se dizer, portanto que a amostra desse estudo é representativa dos idosos que residem nos bairros selecionados, já que foram excluídos os demais. Além disso, foram os idosos que se voluntariaram a participar. No futuro, espera-se que sejam feitos cálculos sobre o grau de representatividade na população desses bairros.

Outra limitação desse estudo é a ausência de variáveis outras que poderiam ter afetado depressão e a própria convivência com eventos estressantes, tais como número de doenças somáticas e funcionalidade. Sendo a CES-D um instrumento que inclui sintomas somáticos, seria importante controlar o efeito de doenças somáticas na amostra estudada, através de diagnósticos clínicos de saúde física.
A funcionalidade, expressa em termos de capacidade para realização de atividades de vida diária, é outro aspecto que pode interferir nos processos de estresse e depressão. Futuros trabalhos devem considerar essas variáveis como fatores intervenientes dos processos de estresse e da manifestação de sintomas depressivos na velhice. Em termos metodológicos, sugere-se a construção de um modelo de base matemática sobre a relação entre as variáveis investigadas, utilizando métodos estatísticos de Path Anaysis, que permitem analisar o peso relativo das variáveis sobre depressão.

Quanto às características da amostra estudada, é importante ressaltar que existe uma menor variabilidade em relação a escolaridade e renda do que a encontrada na população idosa brasileira. Cerca de cinqüenta por cento dos idosos são alfabetizados ou concluíram o nível primário de escolaridade. Somente $3 \%$ dos idosos nunca freqüentaram a escola, enquanto $10 \%$ têm curso superior. A distribuição de renda entre os idosos dessa amostra foi equivalente nas quatro faixas salariais consideradas, o que representa índices contrastantes com aqueles encontrados na população brasileira, sendo considerados obstáculos à generalização dos resultados obtidos neste estudo para população do Brasil. Estudos estatísticos posteriores devem aquilatar a representatividade da amostra do PEN$\mathrm{SA}$, através de métodos estatísticos que verifiquem a validade externa da amostra.

Futuros trabalhos deverão avaliar essas relações em diferentes coortes, para verificar se esta é uma tendência do envelhecimento ou do processo de enfrentamento em geral. Sugere-se a adoção de semelhantes metodologia e embasamento teórico, para que os resultados possam vir a ser comparáveis entre si. Futuros trabalhos poderão avaliar a evolução das variáveis de estresse e depressão, a fim de averiguar se os resultados obtidos neste estudo referem-se a características do processo de envelhecimento ou a aspectos ligados à coorte estudada. É possível que outras variáveis sejam incluídas em futuras análises, para verificar com maior precisão o peso relativo das variáveis investigadas neste estudo sobre a manifestação de sintomas depressivos.

\section{Referências}

Aldwin, C. M. (1990). The Elders Life Inventory (ELsi): Egocentric and nonegocentric stress. In M. A. P. Stephens, S. E. Hobfoll, J. H. Crowther \& D. L. Tennenbaum (Eds.), Stress and coping in late life families. New York: Hemisphere.

Aldwin, C. M. (1991). Does age affect the stress and coping process? Implications of age differences in perceived control. Journal of Gerontology, 46, 171-180.

Aldwin, C. M. (1994). Stress, coping and development: An integrative perspective. New York: Guilford.

Aldwin, C. M., \& Gilmer, D. F. (2004). Health, illness, and optimal aging: Biological and psychological perspectives. Los Angeles, CA: Sage.

Aldwin, C. M., Sutton, K. J., \& Lachman, M. (1996). The development of coping resources in adulthood. Journal of Personality, 64, 837-871. 
Almeida, O. P., \& Almeida, S. A. (1999). Confiabilidade da versão brasileira da escala de depressão em geriatria. Arquivos de Neuropsiquiatria, 57(2-B), 421-426.

Aneshensel, C. S. (1992). Social stress: Theory and research. Annual Review of Sociology, 18, 15-38.

Bandura, A. (1977). Self-efficacy: Toward a unifying theory of behavioral change. Psychological Revierc, 84, 191-215.

Bandura, A. (1982). Self-efficacy mechanism in human agency. American Psychologist, 37, 122-147.

Bandura, A. (1986). Social foundations of thought and action. A social cognitive approach._Englewood Cliffs, NJ: Prentice Hall.

Bandura, A. (1995). Exercise of personal and collective efficacy in changing societies. In A. Bandura (Ed.), Self-efficacy in changing societies. New York: Cambridge University Press.

Bandura, A. (1997). Self-efficacy. The exercise of control. New York: W. H. Freeman.

Barefoot, J. C., Mortensen, E. L., Helms, M. J., Avlund, K., \& Schroll, M. (2001). A longitudinal study of gender differences in depressive symptoms from age 50 to 80 . Psychology and Aging, 16, 342-345.

Beckett, M., Goldman, N., Weinstein, M., Lin, I. F., \& Chuang, Y. L. (2002). Social environment, life challenge and health among the elderly in Taiwan. Social Science and Medicine, 55(2), 191-209

Brown, G. W., \& Harris, T. (1978). Social origins of depression: $A$ study of psychiatric disorder in women. London: Tavistock.

Carstensen, L. L., Gross, J. J., \& Fung, H. H. (1998). The social context of emotional experience. In Annual Review of Gerontology and Geriatrics: Vol. 17. Focus on Emotion and Adult Development. Philadelphia, PA: Springer.

Chapleski, E. E., Kaczynski, R., Gerbi, S. A., \& Lichtenberg, P. A. (2004). American Indian elders and depression: Short- and long-term effects of life events. Journal of Applied Gerontology, 23, 40-57.

Davies, A. D. M. (1996). Life event, health, adaptation and social support in the clinical psychology of late life. In R. T. Wood (Ed.), Handbook of the Clinical Psychology of Aging. New York: Wiley

Folkman, S. (1992). Commentary to part three: Improving coping assessment: Reply to Stone and Kennedy-More. In H. S. Friedman (Ed.), Hostility, coping; and health (pp. 215-223). Washington, DC: American Psychological Association.

Folkman, S., \& Lazarus R. S. (1980). An analysis of coping in middle-aged community sample. Journal of Health Social Behavior, 21, 219-239.

Folkman, S., Lazarus, R. S., Pimley, S., \& Novacek, J. (1987). Age differences in stress and coping processes. Psycology and Aging, 2, 171-184.

Holahan, C. K., \& Holahan, C. J. (1987). Life stress, hassles, and self-efficacy in aging: A replication and extension. Journal of Applied Social Psychology, 17(6), 574-592.

Hughes, D. C., Blazer, D. G., \& George, L. K. (1988). Age differences in life events: A multivariate controlled analysis. International Journal of Aging and Human Development, 27(3), 207-220.

Instituto Brasileiro de Geografia e Estatística. (Ed.). (2000). Censo demográfico. Rio de Janeiro, RJ: Autor.

Karademas, E. C., \& Kalantzi-Azizi, A. (2003). The stress process, self-efficacy expectations, and psychological health. Personality and Individual Differences, 37, 1033-1043

Kavanagh, D. (1992). Self-efficacy and depression. In R. Schwarzer (Ed.), Self-efficacy: Thought control of action (pp. 177-194). Washington, DC: Hemisphere.
Krause, N. (1986). Stress and sex differences in depressive symptoms among older adults. Journal of Gerontology, 6, 727731.

Lazarus, R. S., \& Folkman, S. (1984). Stress, appraisal and coping: New York: Springer.

Lazarus, R. S., \& Folkman, S. (1987). Transactional theory and research on emotions and coping. European Journal of Personality, 1, 141-169.

Martire, L. M., Stephens, M. A. P., \& Townsend, A. L. (2000). Centrality of women's multiple roles: Beneficial and detrimental consequences for psychological well-being. Psychology and Aging, 15(1), 148-156.

Mirowsky, J, \& Ross, C. E. (1995). Sex differences in distress: Real or artifact? American Sociological Review, 60, 449-468.

Neugarten, B. L. (1968). Adult personality: Toward a psychology of the life cycle. In B. L. Neugarten (Ed.), Middle age and aging: A reader in social psychology (pp. 137-147). Chicago, IL: University of Chicago Press.

O'Leary, A. (1992). Self-efficacy and health: Behavioral and stress-physiological mediation. Cognitive therapy and research, 16, 229-245

Pereira, J. C. R. (1999). Análise de dados qualitativos: Estratégias metodológicas para as Ciências da Saúde, Humanas e Sociais. São Paulo, SP: Edusp.

Radloff, L.S. (1977). The CES-D scale: A self-report depression scale for research in the general population. Applied Psychological Measurement, 1, 385-401.

Rodin, J. (1986). Health, control and aging. In M. M. Baltes \& P. B. Baltes (Eds.). The psychology of control and aging (pp. 139165). Hillsdale, NJ: Lawrence Erlbaum.

Seligman, M. E. P. (1977). Desamparo: Sobre depressão, desenvolvimento e morte (M. T. A. Silva, Trad.). São Paulo, SP: Edusp. Stone, A. A., Helder, L., \& Schneider, M. S. (1988). Coping with stressful events: Coping dimentions and issues. In L. H. Coehn (Ed.), Life events and psychological functioning: Theoretical and methodological issues (pp. 182-210). Newburry Park, CA: Sage.

Tavares, S. S. (2004). Sintomas depressivos entre idosos: Relações com classe, mobilidade e suporte social percebidos e experiência de eventos estressantes. Dissertação de Mestrado não-publicada, Faculdade de Educação, Universidade Estadual de Campinas, SP

Tavares-Batistoni, S. S., Neri, A. L., \& Cupertino, A. P. F. B. (2006). Depressive symptoms among aged Brazilians: Psychometric characteristics of the Center for Epidemiological Studies Depression Scale. Manuscrito em preparação.

Vogt, W. P. (1993). Dictionary of Statistics and Metodology: A nontechnical guide for the Social Sciences. London: Sage.

Yesavage, J., Brink, T. L., Rose, T., Lum, O., Huang, O., Adey, V., \& Leirer, V. (1983). Development and validation of a geriatric depression screening scale: A preliminary report. Journal of Psychiatric Research, 17, 37-49.

Young, J. E., Beck, A. T., \& Weinberg, A. (1999). Depressão. In D. H. Barlow (Ed.), Manual clínico de transtornos psicológicos. Porto Alegre, RS: Artes Médicas. 